\title{
Surgical treatment of celiomesenteric trunk aneurysm-7 case report
}

\author{
Chunxi Wang ${ }^{\mathrm{a}, *}$, Xiangjun Cai ${ }^{\mathrm{b}}$, Faqi Liang ${ }^{\mathrm{a}}$, Futao Chu $^{\mathrm{a}}$, Gang Chen ${ }^{\mathrm{a}}$ and Zhiquan Duan ${ }^{\mathrm{c}}$ \\ ${ }^{a}$ Department of General Surgery, Clinical Division of Surgery, Chinese PLA (People's Liberation \\ Army) General Hospital, Beijing 100853, China \\ ${ }^{b}$ Department of Vascular Surgery, the First Teaching Hospital, China Medical University, Shenyang \\ 110001, China \\ ${ }^{c}$ Department of Vascular Surgery, the 202 Hospital of Chinese People's Liberation Army, Shenyang \\ 110002, China
}

\begin{abstract}
The celiomesenteric trunk is a rare anomaly characterized by a common origin of the celiac axis and superior mesenteric artery from the aorta, which accounts for less than $1 \%$ of all celiac artery anomalies, so the aneurysm occurred in such trunk is even rarer. There have been few reports on how to diagnose and deal with such malformed celiomesenteric trunk aneurysms till now. This paper tries to summarize the experience of how to expose and excise such kind of aneurysm according to the seven cases' data. The clinic data were collected retrospectively. There were seven cases with celiomesenteric trunk aneurysm from February 2000 to February 2013, including 5 males and 2 females aged 35 62. The operations were done including aneurysm resection and vascular reconstruction under general anesthesia. The operated patients were followed-up at the sixth month and each year post operation. The vascular stomas were detected or examined by Color Doppler Sonography, spiral Computed Tomography angiography (SCTA). The seven operated patients were cured and discharged from hospital, and they were followed up for 3 10 years (mean time 5 years), with four patients being followed up longer than 5 years. No sign of intestinal ischemia or hepatic ischemia or splenic ischemia was found, and no image of anastomosis stricture or stenosis was found during the follow-up. Five patients are alive now while two patients were dead, with one dying of large area myocardial infarction unexpectedly at 6 years post operation and the other dying of cerebral infarction abruptly at 4 years post operation. It is an effective and safe method to treat the celiomesenteric trunk aneurysm by using by-pass operation with artificial blood vessels, originating from inferior kidney aorta to visceral arteries including hepatic artery, splenic artery and superior mesenteric artery. Its short-term and middle-term effects are relatively better.
\end{abstract}

Keywords: Celiomesenteric trunk, superior mesenteric artery, celiac trunk, aneurysm, operation

\section{Introduction}

A common celiomesenteric trunk is a strange anatomic variation when the celiac and the superior mesenteric arteries originate from a single common trunk, which means that such above arteries come from the same point of the abdominal aorta. Because of its rarity, few lesions of the celiomesenteric

\footnotetext{
${ }^{*}$ Corresponding author: Chun-xi Wang, Department of General Surgery, Clinical Division of Surgery, Chinese PLA (People's Liberation Army) General Hospital, Beijing 100853, China. Tel.: 86-10-66936538; Fax: 86-10-66936538; E-mail: wangchunxi301pw@sina.com.
}

0959-2989/14/\$27.50 @ 2014 - IOS Press and the authors. 
trunk have been reported [1,2]. Although the visceral aneurysms were sometimes reported [3-5], it is very rare to report the malformed celiomesenteric trunk aneurysm as it only accounts for less than $1 \%$ of all celiac artery anomalies [6]. The malformed celiomesenteric aneurysm contains an odd structure which makes hepatic artery, splenic artery, left gastric artery and mesenteric artery come from the same aneurysm rather than from aorta or celiac trunk. Therefore, such special anatomic structure makes therapeutic procedure complicated. Surgical intervention for this condition is challenging $[5,6]$ when the aneurysm is larger or almost occupies the whole trunk, especially in the back of pancreas. The reason is that the hepatic artery, splenic artery, mesenteric artery, and left gastric artery have to be reconstructed once the malformed aneurysm has been cut off. Nevertheless, no successful case with such kind of aneurysm was reported by using endovascular intervention merely and few cases receiving traditional operation have been recorded till now. The seven cases with such kind of the malformed aneurysms from February 2000 to February 2013 are collected and reported as follows.

\section{Clinic data}

\subsection{Patients}

The seven patients with malformed celiomesenteric trunk aneurysm include 5 male and 2 female aged 35 62, with the average age of $40.86 \pm 16.16$ years old. All patients had epigastria discomfort or mildly abdominal pain, and one case had right epigastric pain coexisted with lumbago. There was epigastria tenderness without abdominal rigidity in all cases. The abdominal masses were palpable in four patients whose pulsate with radial pulses coincidently, and the vascular murmurs were heard.

\subsection{Imaging data}

Celiomesenteric trunk aneurysms were demonstrated by color Doppler ultrasonography, computer tomography angiography (CTA) and confirmed by digital subtraction angiography (DSA). The saccular aneurysms with four branches in such malformed portion were found by imaging inspection (including color Doppler ultrasonography, computer tomography angiography and digital subtraction angiography) in all patients(Figures 1 and 2). Such above branches were hepatic artery, splenic artery,

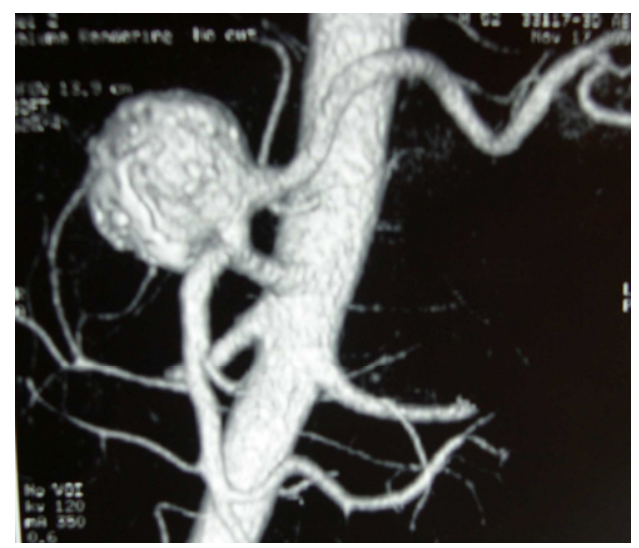

Fig. 1. Celiomesenteric trunk aneurysms (CTA).

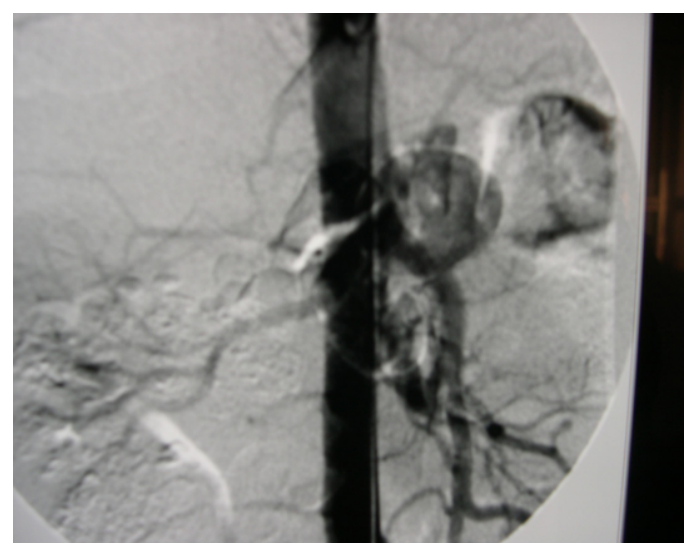

Fig. 2. Celiomesenteric trunk aneurysms (DSA). 
the left gastric artery and mesenteric artery $[7,8]$. The sizes of aneurysms were from 4.8 centimeter to 8 centimeter with mean $5.83 \pm 1.01$ centimeter, shaped as ellipse or fusiform with mural thrombosis and spotted calcification in the aneurysm wall.

\subsection{Operation procedure}

All patients received general anesthesia with tracheal intubation. The extensive exploratory was made thoroughly. Most of the small intestines were put outside from abnormal cavity and were put into the proper transparent plastic bag just beside the incision, which help expose retroperitoneal organs easily and observe the intestinal blood supply in time. When the gastrocolic ligament was opened with electric knife, the regional aorta above the superior pancreatic border was exposed. In order to better control celiac blood supply, the aorta was circled with $8 \mathrm{~F}$ silica gel tube. The superior abdominal aorta was exposed after the pancreas was downward, where the manipulation made the incipient portion of the celiac trunk or the neck of aneurysm exposed extensively. As most of aneurysm body was behind the pancreas, the pancreas has to be put away for better aneurysm exposure. Firstly, the corpus and caudal of pancreas were dissected and separated from the aneurysm; Secondly, the corpus of pancreas was surrounded with silica gel tube which made the operative space relatively spacious; and then the main body of aneurysm was disassociated from periphery tissue with an electric knife, and the four branches efferent from aneurysm were surrounded by $8 \mathrm{~F}$ silica gel tubes respectively (Figure 3). Finally, such malformed aneurysms were ablated post incipient portion of such celiac trunk and four branches were blocked.

The special prepared trifurcate vascular prosthesis was selected for anastomosis. A $1 \sim 2 \mathrm{~cm}$ incision was made in the clamped area and then the end-side anastomosis was made between the top (or beginning end) of the prosthesis and the infrarenal aorta (Figure 4). When the three branches in the distal end of such prosthesis were prepared, the anastomoses were done between the three branches of the vascular prosthesis and the three remained celiac arteries including aorta-hepatic artery, aorta-splenic artery and aorta-superior mesenteric artery one by one (Figures 5 and 6). Therefore, the arterial blood was supplied to such above celiac arteries from aorta through such transplanted vascular prosthesis. As the left gastric artery alone does not lead to gastric ischemia, the seven left gastric arteries are not reconstructed in all cases.

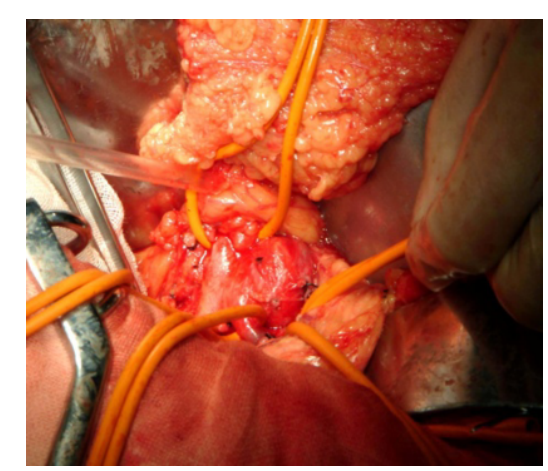

Fig. 3. The four branches efferent from aneurysm were surrounded by $8 \mathrm{~F}$ gel tube.

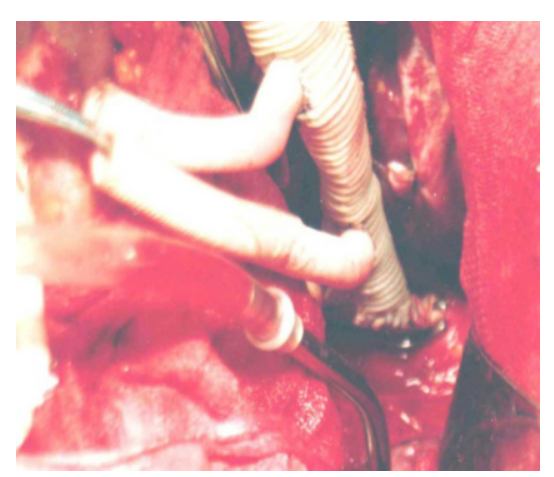

Fig. 4. The anastomosis was made between prepared trifurcate vascular prosthesis and infrarenal aorta. 


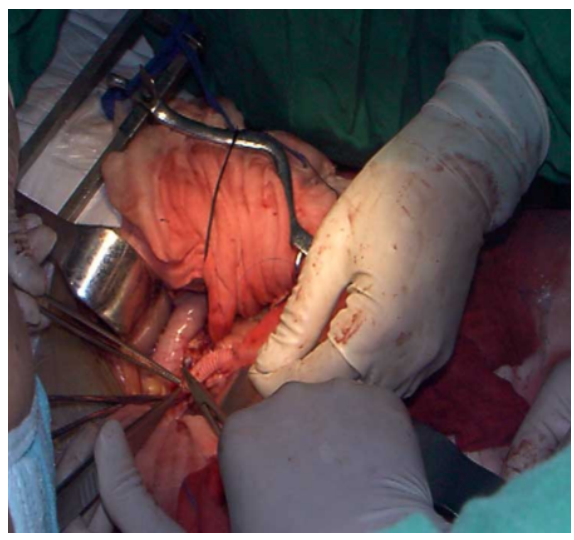

Fig. 5. The anastomosis was made between prosthesis and superior mesenteric artery.

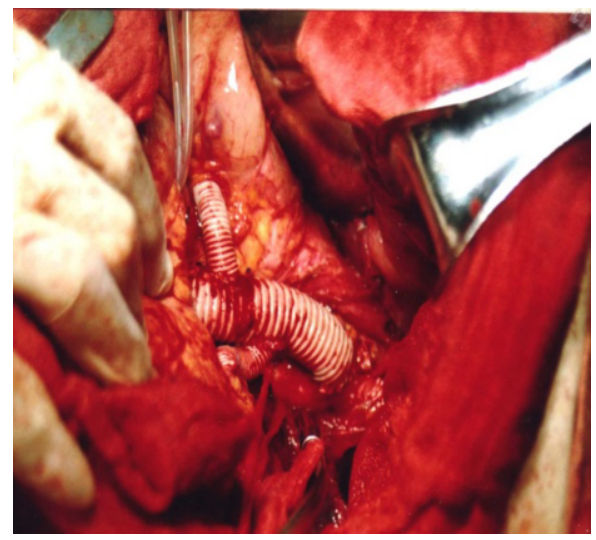

Fig. 6. The anastomosis was made between prosthesis and superior mesenteric artery and hepatic artery.

\section{Results}

The operative mortality was zero. All patients were cured without pancreatitis or lymph leakage or intestinal ischemic complications. All recovered smoothly with the incision healing well and the stitches were taken out on the 7th -9th day post operation. The patients were followed up with color duplex and CTA examination at sixth month and each year post operation. All cases were followed up for 3 10 years (mean time 5 years), with 4 patients being followed up longer than 5 years. No intestinal ischemia or hepatic ischemia sign was found, and no image of anastomosis stricture or stenosis was found during the follow-up. Five patients are alive now and two patients were dead, with one dying of large area myocardial infarction unexpectedly at 6 years post operation and the other dying of cerebral infarction abruptly at 4 years post operation.

\section{Discussion}

It is anomalous that there is a coexistence trunk of superior mesenteric artery and celiac trunk, which is usually called celiomesenteric trunk. It is natural that the celiomesenteric trunk could become stricture or dilated as other celiac arteries [1,2]. It is very rare for an aneurysm to occur in celiomesenteric trunk as the incidence of such coexistence trunk is less than $1 \%$ of celiac arterial deformities, while the celiac trunk and superior mesenteric artery (SMA) are involved in less than 10\% of all visceral aneurysms [5,6,9]. The reason for celiomesenteric trunk aneurysm occurrence is not clear, which might be related to abnormal embryologic development and the arterial degeneration. Unfortunately, most of them were asymptomatic, with few having slight abdominal pain, which makes such patients more dangerous than symptomatic ones as the asymptomatic illness is usually neglected. Sometimes there is an epigastria pain which often radiates to shoulder or back when the aneurysm is being ruptured. Indeed, the most dangerous threaten is that such aneurysm is ruptured abruptly, which usually leads to hemorrhage, shock or death [10-12], with little chance of being rescued due to its abruptness. It is urgent to deal with such illness once the celiomesenteric trunk aneurysm is found.

Although the endovascular skill could deal with a few celiac aneurysms, chiefly in the simple hepatic aneurysm or the splenic aneurysm, sometimes in the superior mesenteric aneurysm [12-14], 
there is no report of success treatment only by using endovascular intervention on the patients with celiomesenteric trunk aneurysm. As the four affiliated branches (i.e., hepatic artery, splenic artery, left gastric artery, SMA) come from the same aneurysm, there is no available or suitable bifurcated stent there or four branches for endovascular intervention presently. The endovascular intervention, for the time being, is not able to deal with such problem. It would be possible to repair the aneurysm and to restore such above branches (at least including hepatic artery, splenic artery, SMA) only if the available qualified bifurcated covered stents occur, and it would be a catastrophe only to keep one branch uncovered let along the others whether to be covered or not. Therefore, the open operation becomes a reasonable management. The technique used to repair the aneurysm depends exclusively on the location, the size, and the shape of the lesion. Occasionally, the aneurysmorhaphy or dissection with patch angioplasty may be feasible if the aneurysm is a very smaller sacular one. As most of the patients have complicated and larger aneurysm, the placement of a graft is usually selected, which includes orthotopic transplantation and by-pass transplantation with artificial blood vessels. The by-pass transplantation is often performed as the celiomesenteric trunk aneurysm is relatively larger and complicated. Therefore, the open operation (including the aneurysm excision, aortic-superior mesenteric artery bypass, aortic-hepatic artery bypass, aortic-splenic artery bypass) actually becomes the chief therapy at present [15]. However, it is very difficult to do such operation as most of aneurysm body hides behind pancreas and next to stomach, spleen and other retroperitoneal organs $[5,6,16]$. To ensure the vascular reconstruction safety, the aneurysm neck is exposed and controlled effectively. The authors' experience is as follows: first, dissociate pancreas body and pull it away downward; second, dissociate aorta above celiac artery and surround the $8 \mathrm{~F}$ silica gel tubes for better controlling blood loss; and then dissect the incipient portion of the celiac trunk as possible as we could. The operative field would become wider after the pancreas was hauled upward or downward, thus the aneurysm could be excised easier. After the four branches were dissected, their incipient portions were surrounded by silica gel tubes. Then the aneurysm was ablated under completely blocked aneurysm neck and such above four branches. Obviously, aorta-viscera by-pass operation is a correct therapy after such aneurysm is resected. The site of aorta stoma was selected on the infrarenal aorta, which could provide better operative field and could avoid renal ischemia.

Generally speaking, only one stoma at aorta site was needed because the distal part of vascular prosthesis may be connected with several branches through end-side anastomosis, which bifurcate vascular prosthesis provided aorta is successfully communicated with several visceral arteries simultaneously. However, it will be permissive to reserve relatively normal arterial wall, or to repair with auto venous patch if the aneurysm encroaches little wall in some branches, which diminishes the chance of regional thrombosis. One patient in our data used such operative method in superior mesenteric artery. As the stomach has four chief arteries to supply blood, there is no obvious gastric ischemia even if anyone branch is ligated or cut off, which is identified by many clinic data. So the left gastric artery might be ligated without reconstruction. No one suffered from gastric ischemia after the left gastric artery is ligated in our follow-up data. Although the operative procedure is complicated, the results show relatively better effects. Like most scholars $[6,15,16]$, the authors presume the traditional operation will still be a chief treatment at least for the time being.

\section{References}

[1] P.H. Connolly, A.J. Meltzer, K.A. Gallagher and R. Dayal, A visceral aortic branch anomaly presenting with a common celiomesenteric-renal trunk, J. Vasc. Surg. 57 (2013), 1675-1677. 
[2] J.M. Petscavage and P. Maldjian, Celiomesenteric trunk: Two variants of a rare anomaly, Australas. Radiol. Suppl. 51 (2007), B306-309.

[3] R. Pulli, W. Dorigo, N. Troisi, G. Pratesi, A.A. Innocenti and C. Pratesi, Surgical treatment of visceral artery aneurysms: A 25-year experience, J. Vasc. Surg. 48 (2008), 334-342.

[4] P. Popov, S. Boskovic, D. Sagic, B. Radevic, N. Ilijevski, D. Nenezic, N. Tasic, L. Davidovic and D. Radak, Treatment of visceral artery aneurysms: retrospective study of 35 cases, Vasa. 36 (2007), 191-198.

[5] H. Higashiyama, K. Yamagami, K. Fujimoto, T. Koshiba, K. Kumada and M. Yamamoto, Open surgical repair using a reimplantation technique for a large celiac artery aneurysm anomalously arising from the celiomesenteric trunk, J. Vasc. Surg. 54 (2011), 1805-1807.

[6] H. Obara, K. Matsumoto, N. Fujimura, S. Ono, T. Hattori and Y. Kitagawa, Reconstructive surgery for a fusiform common celiomesenteric trunk aneurysm and coexistent abdominal aortic aneurysm: Report of a case, Surg. Today 39 (2009), 55-58.

[7] M. Koganemaru, T. Abe, M. Nonoshita, R. Iwamoto, M. Kusumoto, A. Kuharaand and T. Kugiyama, Follow-up of true visceral artery aneurysm after coil embolization by three-dimensional contrast-enhanced MR angiography, Diagn. Interv. Radiol. 20 (2014), 129-135.

[8] R. Krishnan, K. Murali, R. Madan and G. Francis, CT imaging findings and endovascular management of isolated spontaneous dissecting aneurysm of celiac artery, Indian J. Radiol. Imaging 23 (2013), 234-237.

[9] H. Matsuda, H. Ogino, A. Ito, H. Sasaki, K. Minatoya, M. Higashi, T. Yagihara and S. Kitamura, Aneurysm of the celiac artery arising from a celiomesenteric trunk, J. Vasc. Surg. 44 (2006), 660-663.

[10] E. Mammano, M. Cosci, A. Zanon, G. Picchi, E. Tessari, P. Pilati and D. Nitti, Celiomesenteric trunk aneurysm, Ann. Vasc. Surg. 23 (2009), e7-10.

[11] Y. Kanaoka, H. Kubo, The six-year results of a combined surgical and endovascular repair for thoracoabdominal aortic aneurysm involving the visceral arteries, Minerva. Chir. 63 (2008), 229-235.

[12] G. Vitale, F. Simoncini, U.M. Bracale and G. Bergamaschi, Endovascular management of a giant celiac artery aneurysm, Intern. Med. 51 (2012), 671-672.

[13] G.L. Jenssen, J. Wirsching and G. Pedersen, Treatment of a hepatic artery aneurysm by endovascular stent-grafting, Cardiovasc. Intervent. Radiol. 30 (2007), 523-525.

[14] K.O. Karabay, G. Geceer and R. Erdim, Celiac artery aneurysm, Med. Arh. 66 (2012), 283-284.

[15] M.Z. Ghariani, Y. Georg, C. Ramirez, E. Lebied, J. Gaudric, L. Chiche, E. Kieffer and F. Koskas, Long-term results of surgical treatment of aneurysms of digestive arteries, Ann. Vasc. Surg. 27 (2013), 954-958.

[16] C. Twine, A. Wood and I. Williams, Surgical repair of a coeliac artery aneurysm, J. Vasc. Surg. 57 (2013), 848-849. 